\title{
Cooperative Management of Wireless Mesh Networks
}

\author{
George Athanasiou \\ KTH Royal Institue of Technology, Sweden, \\ E-mail: georgioa@kth.se
}

\begin{abstract}
The association/handoff procedures are important components in a balanced operation of 802.11-based wireless mesh networks. These networks are usually consisted of a static wireless backbone. The peripheral routers serve as access points (APs) and are associated with the stations (STAs) that are distributed in the network. Due to the large number of mesh APs, the STAs must decide to be associated with the mesh APs that will provide them the best QoS. In this paper we introduce the concept of cooperative association, where the STAs can share useful information in order to improve the performance of the association/handoff procedures. Furthermore, we introduce a load balancing mechanism that can control the communication load of each mesh AP in a distributed manner. We evaluate the performance of our mechanisms through OPNET simulations.
\end{abstract}

\section{INTRODUCTION}

The IEEE 802.11 [19] wireless local area networks (WLANs) were originally designed to give a solution to the huge problem of tangled cables of the end user devices. The stations (STAs) are wirelessly connected to the available access points (APs) and the APs are connected to a wired backbone network. The evolution of these networks are the mesh networks where a wireless backbone network is set up in order to support end-to-end wireless user communication [17]. Several wireless routers that are part of this wireless backbone network, forward the traffic in the network. In addition a number of these routers also serve as APs. The STAs are associated with the available APs and send their data through them. Undoubtedly, the mesh networks are quite similar to the infrastructure WLANs but they take an important benefit of their self-organized structure and their dynamic nature. We can allegate that the mesh networks are ad-hoc networks that operate in an infrastructure mode. In this way they combine the benefits of ad-hoc networks and WLANs.

In this paper we propose a cooperative association concept that speeds up the basic association procedure. It is independent from the association protocol that is active in the network. The main outcome of this mechanism is that it eliminates the delays due to scanning/probe and reassociation phases. The algorithm is called "cooperative", since nodes share their view of the network with each other. Furthermore, in this work we present a novel load balancing scheme. Wireless mesh networks can usually be overloaded due to the large number of the associated STAs that try to send their data or due to the large communication traffic that must be routed in the mesh backbone. Our mechanism tries to overcome these situations by controlling the communication load in a distributed manner. The APs measure the load conditions in their neighborhood and inform their associated STAs. The STAs execute a heuristic algorithm in order to optimize their communication. Our load balancing mechanism uses crosslayer information in order to provide a balanced MAC and

This work was supported by the Swedish Research Council and the VR project of C. Fischione: "In Network Optimization"
Routing layer operation. We would like to notice that this is a preliminary work, with the potentiality to important developments in near future.

The rest of the paper is organized as follows. In section II we present the state of the art and briefly describe the main restrictions in "expanding" the network capabilities that are introduced by the current 802.11 association scheme. Section III presents the application of a cross-layer association scheme in wireless mesh networks that was proposed in [1]. In section IV, we consider the cooperative association mechanism. In section $\mathrm{V}$, we describe a load balancing mechanism that guarantee a jointly MAC/Routing balanced operation. Section VI presents the evaluation of the proposed mechanisms. Finally, section VII concludes the discussion.

\section{ASSOCIATION AND LOAD BALANCING SCHEMES}

IEEE 802.11 defines an association procedure based on the RSSRI (Received Signal Strength Report Indicator). The unassociated STAs or the STAs that are trying to reassociate with a new AP, initialize a scanning process to find the available APs that are placed nearby. They measure the RSSRI values of each $\mathrm{AP}$ and associate with the AP that has the highest RSSRI value (the strongest received signal). Several studies have proved that the RSSRI-based association mechanism can lead to poor network performance while the networks resources are not utilized efficiently [5], [6]. Therefore the research community focused their research interests in designing new association methodologies that will provide better resource utilization in the network. In [1] the authors propose new dynamic association and reassociation procedures that use the notion of the airtime cost in making association decisions. In [2], the authors study a new STA association policy that guarantees networkwide max-min fair bandwidth allocation in the network. In [3], the authors propose an association scheme that takes into account the channel conditions (the channel information is implicitly provided by $802.11 \mathrm{~h}$ [21] specifications). The work in [4] proposes an improved client association and a fair resource sharing policy in 802.11 wireless networks. The authors in [5], propose a technique to eliminate the probe phase delay of the association process. The system presented in [6] ensures fairness and QoS provisioning in WLANs with multiple APs. Management frame synchronization is the basic part in the proposed mechanism presented in [7]. In [8], the authors formulate the association problem using neighbor and non-overlap graphs. In [9], multiple radios are used in order to implement more effective handoff mechanisms. In [10] the problem of optimal user association to the available APs is formulated as a utility maximization problem. The work in [11] proposes a new mechanism where the traffic is split among the available APs in the network and the throughput is maximized by constructing a fluid model of user population that is multi-homed by the available APs in the network. 
Finally, in [12] there is an interesting study of different fast handoff mechanisms.

Recently, there has been some work investigating the load balancing problem in WLANs. In [13] the authors discuss some load balancing approaches and how the future $802.11 \mathrm{k}$ [22] functionalities could contribute in implementing these mechanisms in 802.11 WLANs. The work in [14] proposes a cross-layer framework where packet level scheduling, handoff and system-level load balancing are jointly performed. The techniques presented in [15] control in an intelligent manner the cell size of congested APs (cell-breathing), achieving in that way a load balanced network operation. Last but not least, the work in [16] proposes a new cell-breathing scheme that act as a load balancing mechanism to control the congested cells in a WLAN.

The work in [1] introduces a cross-layer association framework that works in the direction of optimizing the association decisions of the STAs in the network. A STA that is initiating a reassociation process has to scan for available APs in its' neighborhood. This scanning is a time consuming process and slows down the handover. In order to eliminate these delays we propose a new association concept where we support STA collaboration. Furthermore, in the cross-layer mechanisms we have observed especially in high load conditions that the mesh APs that provide good QoS are usually overloaded. Communication overload is quite often in pure 802.11 operation too, even in low load conditions. Consequently, the proposed load balancing scheme tries to cope with this problem and provide a balanced network operation.

\section{ApPlying Cross-Layer Association in 802.11-BASED MESH NETWORKS}

In this section we provide a high level overview of the crosslayer association mechanism that is proposed in [1] which is the basis of the proposed mechanisms in this paper.

The proposed end-to-end QoS aware cross-layer association scheme is designed for 802.11-based mesh networks. In this sophisticated mechanism the association decision is based on a new metric called airtime cost that reflects the average duration for which the channel is occupied. The authors define the airtime cost of station $i \in U_{a}$, where $U_{a}$ is the set of stations associated with AP $a$, as:

$$
C_{a}^{i}=\left[O_{c a}+O_{p}+\frac{B_{t}}{r^{i}}\right] \frac{1}{1-e_{p t}^{i}} .
$$

In (1), $O_{c a}$ is the channel access overhead, $O_{p}$ is the protocol overhead and $B_{t}$ is the number of bits in the test frame. Some representative values (in $802.11 \mathrm{~b}$ networks) for these constants are: $O_{c a}=335 \mu \mathrm{s}, O_{p}=364 \mu \mathrm{s}$ and $B_{t}=8224 \mathrm{bits}$. The input parameters $r^{i}$ and $e_{p t}$ are the bit rate in $M b s^{-1}$, and the frame error rate for the test frame size $B_{t}$, respectively.

The load on the "uplink" channel of a particular AP $a$ is defined as:

$$
C_{a}^{u p}=\left[O_{c a}+O_{p}+B_{t} \overline{\left(1 / r^{u p}\right)}\right] \frac{1}{1-\overline{e_{p t}^{u p}}}\left|U_{a}\right|,
$$

where $\overline{e_{p t}^{u p}}, \overline{r^{u p}}$, and $\left|U_{a}\right|$ are the average uplink error probability, average uplink transmission rate and the number of STAs associated with AP $a$ respectively. The load on the "downlink" is defined as:

$$
C_{a}^{\text {down }}=\left[O_{c a}+O_{p}\right] \sum_{j \in U_{a}} \frac{1}{1-e_{p t}^{j}}+B_{t} \sum_{j \in U_{a}} \frac{1}{r^{j}\left(1-e_{p t}^{j}\right)} .
$$

The main idea of this mechanism is that the STAs base their association decision on the cumulative uplink and downlink airtime cost. Specifically, each AP a broadcasts with its beacons, information about $\overline{e_{p t}^{u p}}, \overline{r^{u p}}$, and $\left|U_{a}\right|$. The STA receives this information and calculates $C_{a}^{u p}$. It also receives $C_{a}^{\text {down }}$ from each AP $a$ and finally selects for association the AP with the minimum $C_{a}^{u p}+C_{a}^{\text {down }}$. In this way the STA can be associated with the less loaded AP that can provide the best communication channel.

In order to apply this mechanism in 802.11-based wireless mesh networks the authors extended the previous mechanism in a cross-layer manner, having in mind the end-to-end QoS provisioning in the network. In a wireless mesh network there is a popularity of mesh APs that are available for association. In order to achieve high QoS provisioning we must consider the end-to-end case where the wireless backhaul network plays an important role.

The upcoming 802.11s [20] standard introduces the airtime link cost as the default routing metric in the mesh backhaule. The Radio Metric-Ad Hoc On Demand Distance Vector (RMAODV) protocol modifies the basic AODV protocol by selecting the end-to-end path with the minimum total airtime cost.

In this mechanism there is a combination of the routing airtime costand the association airtime cost. The total end-toend airtime cost is defined as:

$$
T C_{i}^{r c v}=\left(A C_{i}^{u p}+A C_{i}^{\text {down }}\right) w_{1}+R C_{i}^{r c v} w_{2},
$$

where $T C_{i}^{r c v}$ is the total weighted cost calculated for MAP $i$, $A C_{i}^{u p}, A C_{i}^{\text {down }}$ are the association airtime costs for the uplink and downlink respectively, $R C_{i}^{r c v}$ is the routing airtime cost for the path from MAP $i$ to the receiver $r c v$ and $w_{1}, w_{2}$ are the weights.

The main idea in this end-to-end QoS aware mechanism is that the STAs operate in an active way by sending probe frames to the candidate for association APs. We can call these probe frames "per-receiver association requests" that are used by the STAs to announce their data receiver in order to come up with optimal association decision. The RM-AODV calculates the routing airtime cost from each AP to the data receiver and these values are broadcasted by each candidate AP. The STAs follow the previous procedure in parallel with the calculation of the association airtime cost (using uplink and downlink costs). Finally the association airtime cost and the routing airtime cost are weighted. Each STA calculates $T C_{i}^{r c v}$ and selects for association the mesh AP with the minimum total airtime cost. The previous mechanism can be dynamically executed in a periodic manner by each STA in order to initiate reassociation process in case that a new AP can provide better QoS in the network. An interested reader can refer to [1] for a detailed description of the protocol.

\section{A Cooperative Association Mechanism}

In this section, we introduce a cooperative association mechanism whose goal is to speed up the basic association process. This mechanism is independent from the underlying association decision protocol. However, in this work we jointly use the cooperative mechanism with the cross-layer association mechanism in order to optimize the handoff delay, and thus, the end-to-end delay.

In a wireless mesh network the STAs can cooperate to share their view of the network. In particular, the STAs already associated with the network can relay the information regarding different APs in the network to the STAs not yet associated or the STAs re-associating. By this way, STAs will obtain information about the available APs in an expedited fashion, effectively removing delays due to scanning the channels. 


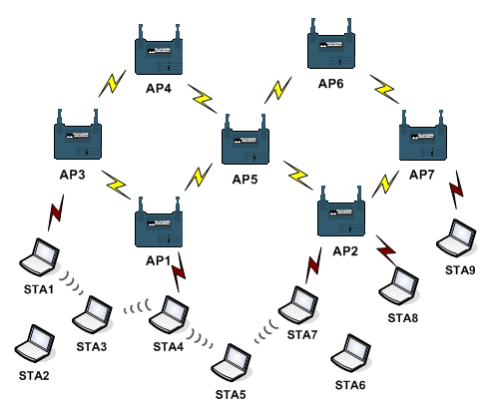

Fig. 1. Cooperative association in a wireless mesh network.

We discuss the basic idea of our cooperative mechanism through the description of an example mesh network scenario. Figure 1 depicts a mesh network where STA1, STA4, STA7, STA8 and STA9 are already associated with the available APs. In this figure we consider a scenario where STA3 and STA5 have just turned on and are initiating an association process in order to associate with an AP. Traditionally these STAs must scan the available channels in order to find an AP for association. The proposed approach aims at eliminating the scanning delay. This can be achieved by introducing cooperation between the wireless STAs. If STA1 and STA4 informs STA3 about the operational frequencies of AP3 and AP1, STA3 does not need to spend time in the scanning phase. Besides, STA1 and STA4 already know the uplink and downlink channel conditions (in the communication with the APs that are currently associated with) and can inform STA3 about these. Consequently STA3 can be aware of the operational frequency and the load of the AP3 and AP1.

The basic component in our cooperative mechanism is a special table that is maintained by each associated STA. This table contains the information about the channel and load conditions of the available APs. It is called ASSOC_TABLE and is regularly updated by the STAs. ASSOC_TABLE is depicted bellow:

\begin{tabular}{|c|c|c|c|}
\hline MAC & CHAN. & LOAD & T/STAMP \\
\hline$M A C(A P 1)$ & 1 & 13 & 123 \\
\hline$M A C(A P 2)$ & 6 & 45 & 134 \\
\hline
\end{tabular}

In the ASSOC_TABLE the load of the APs is represented by their cumulative uplink and downlink airtime cost. We keep a separate timestamp for each table record in order to check the suitability of the stored information.

In the context of our cooperative mechanism the STAs can operate in two modes. In the first mode STAs periodically broadcast their ASSOC_TABLE tables. By this way, the STAs that are in close proximity to each other can keep up to date their channel and load information of the APs in the network. In the second mode of operation, a newcomer STA first sends a control frame to its neighbors in order to acquire the appropriate information. This control frame is an enhanced "CCooperative Probe Request", frame also containing any information related to the association. The neighboring STAs receiving this control frame respond with the broadcast of their ASSOC_TABLE. The newcomer STA builds its own $A S S O C_{-} T A B L E$ according to the information collected from its neighbors. In our simulations, both of these modes are implemented.

Figure 2 depicts the flow chart that is executed when a STA receives the broadcasted ASSOC_TABLE. Firstly the STA checks if there is any entry in the table that contains information for the current AP. In case that there is an entry, the STA updates the corresponding information and otherwise, the STA generates a new entry inside the table.

As we have mentioned, in order to avoid scanning delay, the STAs use the information stored in their ASSOC_TABLE. In case that a STA initiates a scanning or a reassociation process it has to execute the procedure that is depicted in Figure 2. As depicted in Figure 2 for each AP there are two separate checks. First, the suitability of the stored information is checked by comparing the timestamp of the specific record with the current time. In addition we check the load of the current AP. We have introduced a threshold that helps us avoid the overloaded situations in the network. The main idea of this threshold is that in case that an AP is overloaded we must exclude it from the list of the candidate APs for association.

In our proposed cooperative association mechanism, the STAs operate in infrastructure and ad-hoc modes. Infrastructure mode is used to send data through the network and adhoc mode is used to communicate with the neighboring STAs and to relay the ASSOC_TABLE. There are several ways to achieve this behavior in a STA. One is to have the wireless card dynamically changing the mode from infrastructure to ad-hoc, every time the STA needs to execute the cooperative association procedure. Being in this mode, the node can communicate with neighbors that operate in the same channel and exchange information with them about existing APs. An alternative would be the use of a "control channel"" where all the stations would exchange scanning information. It is obvious that in the proposed scheme, the stations still have to execute the scanning procedure in order to update their ASSOC_TABLE. Nevertheless, now the procedure is executed less often, since the stations receive association information from neighbors.

\section{A Sophisticated LoAd BALANCING Mechanism}

In this section we propose a novel load balancing scheme that is based on the airtime information. First, we describe the main idea of the load balancing scheme: In dynamic mesh networks there is a variety of APs that are available for association. Traditionally the RSSRI-based association mechanism measures the channel strength of the available APs and leads the STAs to be associated with the APs that provide the "clearest" or "strongest" channel. Unfortunately this can lead to overloaded situations. In [1] the authors proposed a sophisticated cross-layer association mechanism that tries to phase this problem. Routing is an important component of this association mechanism. The STAs obtain the appropriate information for the uplink and downlink channels and the mesh backhaul in order to optimize their association decision. In our experiments, we have seen that in high load network conditions the performance of the network decreases. There is a demand for a sophisticated mechanism that can balance the load of the network. This mechanism extends the performance bounds of the cross-layer association mechanism by controlling the communication load in an autonomous manner.

In our load balancing scheme the total airtime cost for the mesh AP $i$ in routing the data to the receiver $r c v$ is defined in equation (4). In [1] there is a detailed description of the computation of the total airtime cost. The main idea is that a STA optimizes its' association decision by computing the total airtime costs in their neighbor and associating with the mesh AP that can provide the best QoS (that has the lower total airtime cost). The weights $w_{1}$ and $w_{2}$ play an important 


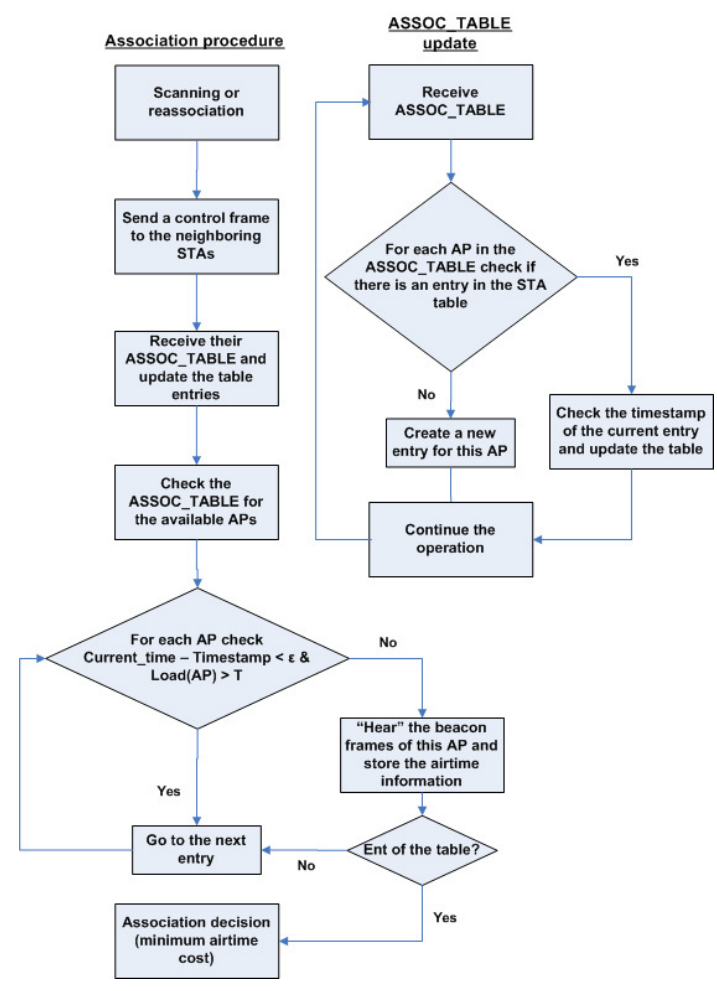

Fig. 2. The main process executed by each STA.

role in the computation of the total airtime cost of a particular data transmission and therefore they determine the association decision of a STA. In our load balancing mechanism we apply a heuristic algorithm to adaptively change these weights "on the fly", according to the communication load of the cells.

We now describe an example communication scenario which shows the importance of a load balancing mechanism. As we mentioned before, an AP in the mesh network can guarantee data routing through efficient routes to a possible receiver (routes with low cumulative routing airtime cost). In case that there is a huge amount of neighboring STAs associated with this AP, it is possible to face a bottleneck at the first, wireless hop of the transmission. In order to overcome these situations, we have to adaptively control the load of the current cell. A cell breathing strategy based on the weight adaptation is executed. By increasing w1 we enforce the impression of the association airtime cost (that reflects the AP load) in the total airtime cost. By this way the STAs that are associated with the current AP will be aware of the communication overload. During the following subsection we describe in detail this mechanism.

We define a balancing index $b$ (introduced in [18]) that reflects the load conditions in a neighbor $n$ ( $n$ neighboring APs) as:

$$
b=\frac{\left(\sum_{i=1}^{n} A C_{i}\right)^{2}}{n \sum_{i=1}^{n} A C_{i}^{2}}
$$

where $A C_{i}=C_{i}^{u p}+C_{i}^{\text {down }}$ for each AP $i$. Each AP $i$ broadcasts its' $b$ value to the neighboring APs in a periodic manner. This broadcasting can be performed using the Local Association Base Advertisement (LABA) mechanism that is proposed in $802.11 \mathrm{~s}$ standard. This mechanism informs the entire mesh network about the STAs associated with the available mesh APs. The mesh APs periodically broadcast LABA messages that contain useful information. We can extend these messages by incorporating in them the airtime information of each AP. Consequently, the mesh APs are aware of the channel communication conditions of each mesh AP in the network.

Considering that we have two neighboring APs we compute the load balancing index:

$$
b=\frac{\left(A C_{1}+A C_{2}\right)^{2}}{2\left(A C_{1}^{2}+A C_{2}^{2}\right)}=\left\{\begin{array}{l}
\left.1, A C_{1}=A C_{2} \text { (balanced }\right) \\
\frac{1}{2}, A C_{1}=0 \text { or } A C_{2}=0 \\
1>b>\frac{1}{2}, \text { otherwise }
\end{array}\right.
$$

Generally for $n$ neighboring APs:

$$
b=\left\{\begin{array}{l}
1, A C_{i}=A C_{j}=\ldots=A C_{k} \text { (balanced) } \\
\frac{1}{n}, A C_{1}=0 \text { or } A C_{2}=0 \text { or } \ldots A C_{n}=0 \\
1>b>\frac{1}{n}, \text { otherwise }
\end{array}\right.
$$

In a neighborhood that contains $n$ APs we introduce a threshold $1>T \geq 1 / n$ which represents the lower bound of a balanced network operation. In case $b<T$ the STAs must increase weight $w_{1}$ in order to make an association decision based mainly on the association airtime cost (AC). The cumulative association airtime cost represents the load of an AP. In our experiments we have seen that in high loaded networks we must chose a threshold close to 1 and in very low load conditions we must chose a threshold close to $1 / n$. We have run different load scenarios in order to choose the appropriate $T$ values.

During the execution of our heuristic algorithm the mesh APs periodically receive the airtime cost values from the neighboring mesh APs and compute the balancing index $b$. The mesh APs broadcast this index in the beacon frames and their associated STAs can "hear" them, extract the carried information and compare $b$ with the predefined threshold. In case $b<T$ there is an unbalanced network operation in this neighborhood, the STAs increase the value of $w_{1}$ and compute the new total airtime cost. At this step of the heuristic algorithm STA reassociations are quite often due to the modification of $w_{1}$.

\section{System EVALUATION}

The proposed mechanisms have been implemented in OPNET [23]. We have built our protocols on top of the IEEE 802.11 standard and in this way we can achieve backward compatibility. We have modified the main control frames (beacon and probe frames) in order to incorporate the appropriate information in them. The light modifications that we have introduced in the basic functionality of the IEEE 802.11 standard do not affect the performance of the network.

\section{A. The Multi-Cell Scenario}

We first study a multi-cell 802.11 network that consists of four overlapping cells. In such simple topologies we can control the parameters of our system and we can have a clear view of the performance of the proposed protocols. The STAs are uniformly distributed in the network and their data frames are transmitted at $1024 \mathrm{kbps}$. We compare the performance of the basic 802.11-based association mechanism, the same mechanism equipped with cooperation between the stations, the airtime association mechanism and the airtime mechanism 


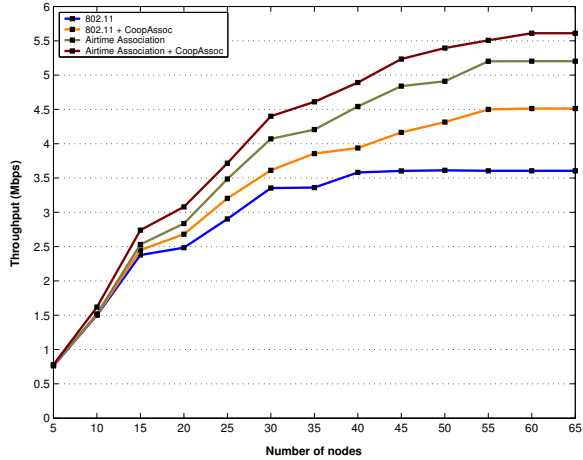

Fig. 3. Throughput (multi-cell scenario).

equipped with STA cooperation, while the communication interference changes during the network operation.

Figure 3 depicts the network throughput while the number of the associated STAs increases. We compare the throughput values that are achieved during the execution of the airtime association mechanism, the cooperative association mechanism and the basic 802.11-based association scheme. It is clear that the highest throughput values are achieved when we apply jointly the airtime association and the cooperative association mechanism. Contrarily, the 802.11 has the worst performance during our studies. We have to point out that the cooperative mechanism that we have introduced in this work speeds up the association procedure. We jointly apply this mechanism with the airtime and the basic 802.11-based association mechanism.

\section{B. The Mesh Network Scenario}

In order to measure the end-to-end network performance we study the application of the proposed mechanisms in a 802.11-based wireless mesh network. We have simulated a wireless mesh network in the OPNET simulation environment. The wireless routers that are provided by the OPNET wireless module are part of the backhaul network. The peripheral routers serve as APs as well. In the cross-layer mechanisms we need information from the routing layer. Therefore, we have modified the basic AODV routing protocol protocol in order to implement a link quality aware AODV (where the airtime cost serves as an a routing decision metric). In other words we have implemented RM-AODV that is introduced by 802.11 s standard and we have applied this routing protocol at the mesh backhaul. In addition, we have implemented a crosslayer interface which supports the passing of information from one layer to another while the OPNET suite is not equipped with such mechanisms. The STAs are uniformly distributed in the wireless mesh network. For the communication between the wireless routers in the backhaul network, we use the physical model of IEEE 802.11a OFDM physical layer. The supported physical rate of this scheme is 12 Mbps. The STAs that are associated with the available peripheral APs transmit their packets at $1024 \mathrm{kbps}$. We have simulated the cross-layer association, the cooperative association and the load balancing mechanisms by introducing light modifications in the basic functionality of 802.11 (frame modifications, etc.).

We have simulated a VoIP application in the same 802.11based wireless mesh network. In our simulations we have uniformly placed several VoIP clients in the network. We run different simulation scenarios where we vary the number of the VoIP sessions that are supported in parallel.
First of all we measure the average local client access delay in the network. In practice, this delay reflects the time from when the packet is generated until it leaves the client interface. The number of the sessions that are supported in parallel increases from 2 to 24. Figure 4(a) depicts the average VoIP client access delay. The load balancing mechanism (enhanced with cooperation) achieves the lower client access delays in the network. Our load balancing mechanism minimizes the channel access delay while it provides a "cell breathing" to the overloaded cells. The associated STAs are optimally associated in order to maintain a balanced network operation. Consequently, our load balancing mechanism keeps the client access delay in low level while the traditional 802.11 operation overloads the network and the client access delay is continually increased. In high load conditions the delay improvement that is introduced by the load balancing mechanism is quite impressive.

Figure 4(b) depicts the average local VoIP AP access delay in the network. This delay is the time between the arrival of a VoIP packet to the AP until it is ether successfully transmitted over the wireless mesh network or dropped. It is clear that we get the same simulation results with the client access delay. The load balancing mechanism (enhanced with cooperation) has the best performance. In 802.11 the overloaded APs (in high load conditions) have a lot of traffic to forward to the mesh backhaul network. The main consequence is that the VoIP packets have to wait for a long time to be transmitted by the APs, introducing in this way huge AP access delays.

In figure 5(a) we observe the average end-to-end delay in the VoIP packet transmission. The end-to-end delay is affected by the previous two kinds of delays that we have described in detail and the routing delay that is introduced in the backhaul network. The load balancing mechanism (enhanced with cooperation) achieves lower end-to-end delays in the network. Especially in high load network operation the delay improvement is huge. This improvement is true due to the fast VoIP client and AP access in the network, the effective link aware AODV routing protocol in the mesh backhaul and the sophisticated "cell breathing" achieved by the load balancing mechanism in overloaded cells. We argue that the most interesting result is depicted in figure 5(a): The pure 802.11 operation can support at most 14 sessions in parallel while the proposed load balancing mechanism has the capability to support 24 sessions in parallel. Therefore we gain approximately $72,5 \%$ network performance improvement. The network capabilities are expanded by the use of the sophisticated load balancing mechanism.

The last figure (figure 5(b)) depicts the dropped data during the operation of the mesh network. Channel errors, contention and packet collisions are the main reasons for this data dropping. Data dropping in 802.11 is kept in high levels. Our proposed sophisticated mechanisms decrease the data dropping and manage to keep it low even in high load conditions.

\section{CONCLUSIONS}

In this paper we proposed a new association mechanism that introduces cooperation between the STAs in a wireless mesh network. The association process is executed in a cooperative manner in order to eliminate the association/reassociation delays. Furthermore, we proposed a sophisticated load balancing scheme that guarantees a balanced network operation. The heuristic algorithms jointly balance the MAC/Routing communication load. Our future directions include the implementation 


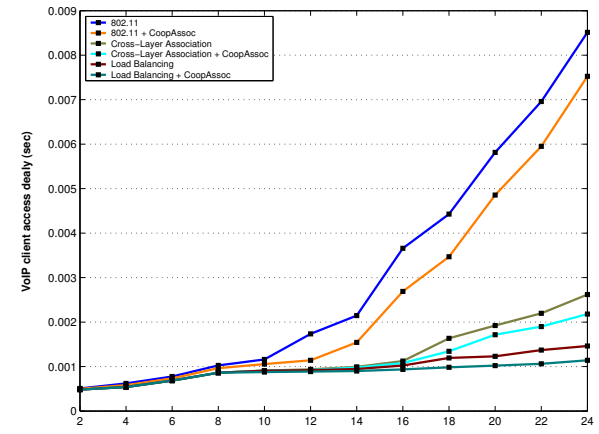

(a) Average client access delay.

Fig. 4. Average VoIP delays.

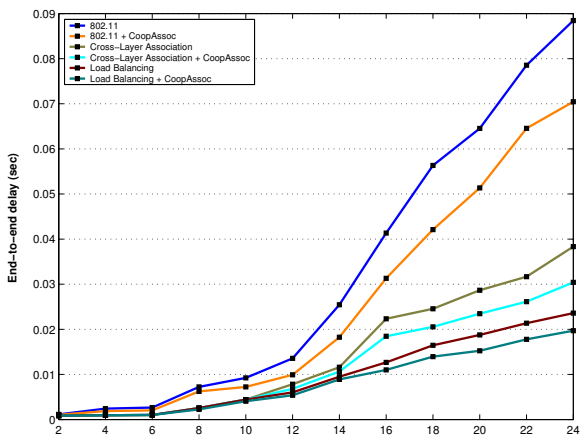

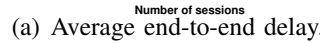

Fig. 5. Average end-to-end delay and dropped data in VoIP.

of these mechanisms using Linux open source drivers and setting up a testbed where we will evaluate our system in real time conditions.

\section{REFERENCES}

[1] G. Athanasiou, T. Korakis, O. Ercetin and L. Tassiulas, "Dynamic Cross-Layer Association in 802.11-based Mesh Networks", To appear in IEEE INFOCOM 2007, Anhcorage, Alaska, USA, May 2007.

[2] Y. Bejerano, S. Han and L. Li, "Fairness and load balancing in wireless lans using association control", In MobiCom, 2004

[3] T. Korakis, O. Ercetin, S. Krishnamurthy, L. Tassiulas, and S. Tripathi, "Link Quality based Association Mechanism in IEEE 802.11 h compliant Wireless LANs", in Workshop on Resource Allocation in Wireless ant Wireless LANs", in Workshop

[4] B. Kauffmann, F. Baccelli, A. Chaintreau, V. Mhatre, K. Papagiannaki and C. Diot, "Measurement-Based Self Organization of Interfering 802.11 Wireless Access Netwroks", To appear in IEEE INFOCOM 2007, Anhcorage, Alaska, USA, May 2007.

[5] W. Arbaugh, A. Mishra and M. Shin, "An empirical analysis of the IEEE 802.11 MAC layer handoff process", ACM SIGCOMM Computer Communication Review, 33, 2003.

[6] Y. Bejerano and R. Bhatia, "Mifi: A framework for fairness and QoS assurance in current IEEE 802.11 networks with multiple access points", In IEEE Infocom, 2004

[7] I. Ramani and S. Savage, "SyncScan: Practical fast handoff for 802.11 infrastructure networks", In INFOCOM '05: The 23rd Conference of the IEEE Communications Society, March 2005

[8] M. Shin, A. Mishra, and W. A. Arbaugh, "Improving the latency of 802.11 hand-offs using neighbor graphs", In MOBISYS, New York, USA, 2004

[9] V. Brik, A. Mishra and S. Banerjee, "Eliminating Handoff Latencies in 802.11 WLANs Using Multiple Radios: Applications, Experience, and Evaluation", in Proceedings of ACM/USENIX Internet Measurement Conference (IMC), October 2005.

[10] A. Kumar and V. Kumar, "Optimal Association of Stations and APs in an IEEE 802.11 WLAN", in Proceedings of of the National Conference on Communications (NCC), IIT Kharagpur, January 2005.

[11] S. Shakkottai, E. Altman and A. Kumar, "The Case for Non-cooperative Multihoming of Users to Access Points in IEEE 802.11 WLANs", in INFOCOM 2006, Barcelona, Spain, April 2006.

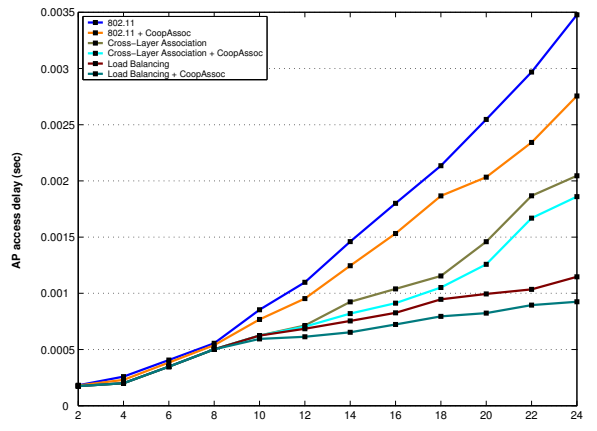

(b) Average AP access delay.

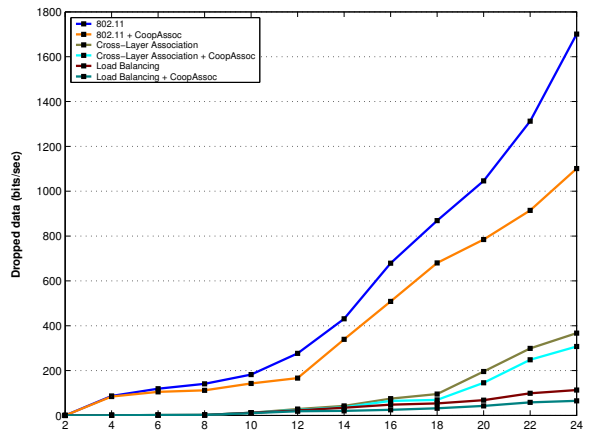

(b) Dropped data. IEEE 802.11 Wireless Networks", in IEEE Communications Surveys \& Tutorials (CST), August 2006.

[13] E.Garcia, R.Vidal, J.Paradells, "Load Balancing in WLANs through IEEE 802.11k Mechanisms", in Proceedings of IEEE Symposium on Computers and Communications, Cagliari, June 2006

[14] A. Sang, X. Wang, M. Madihian and R. D. Gitlin, "Coordinated Load Balancing, Handoff/Cell-site Selection, and Scheduling in Multi-cell Packet Data Systems", in Proceedings of MOBICOM 2004, Philadelphia, Pensylvania, USA, September 2004.

[15] Y. Bejerano and S. Han, "Cell Breathing Techniques for Load Balancing in. Wireless LANs", in INFOCOM 2006, Barcelona, Spain, April 2006.

[16] P. Bahl, M. T. Hajiaghayi, K. Jain, V. Mirrokni, L. Qiu, and A. Saberi, "Cell Breathing in Wireless LANs: Algorithms and Evaluation", in IEEE Transactions on Mobile Computing, Vol 6, February 2007.

[17] I. F. Akyildiz, X. Wang and W. Wang, "Wireless mesh networks: a survey", Computer Networks 47 (2005) 445487.

[18] D. Chiu and R. Jain, "Analysis of the Increase/Decrease Algorithms for Congestion Avoidance in Computer Networks", Journal of Computer Networks and ISDN, Vol. 17, No. 1, June 1989, pp. 1-14

[19] IEEE 802.11: Wireless LAN Medium Access Control (MAC) and Physical Layer (PHY) Specifications, ANSI/IEEE Std 802.11, 1999 Edition.

[20] IEEE 802.11s: Wireless LAN Medium Access Control (MAC) and Physical Layer (PHY) Specifications: Simple Efficient Extensible Mesh (SEE-Mesh) Proposal

[21] IEEE 802.11h: Wireless LAN Medium Access Control (MAC) and Physical Layer (PHY) Specifications: Spectrum and Transmit Power Management Extentions in the $5 \mathrm{GHz}$ Band in Europe

[22] IEEE 802.11 WG. Draft Supplement to Standard for Telecommunications and Information Exchange Between SystemsLAN/MAN Specific RequirementsPart 11: Wireless Medium Access Control (MAC) and Physical Layer (PHY) Specifications: Specification for Radio Resource Measurement, IEEE 802.11k/D3.0. New York, USA: The Institute of Electrical and Electronics Engineers, Inc., October 2005.

[23] “OPNET", http://www.opnet.com, Radio/Wireless Models. 